\title{
Distributed Measurement of Temperature for PCC Energy Pile Using BOFDA
}

\author{
Lei Gao, ${ }^{1}$ Baoquan Ji, ${ }^{1}$ Gangqiang Kong, ${ }^{1}$ Xu Huang, ${ }^{1}$ Mingkun Li, ${ }^{1}$ and Ali H. Mahfouz ${ }^{2,3}$ \\ ${ }^{1}$ Key Laboratory of Geomechanics and Embankment Engineering, Ministry of Education, Hohai University, Nanjing 210098, China \\ ${ }^{2}$ Faculty of Petroleum and Mining Engineering, Suez University, Suez 34721, Egypt \\ ${ }^{3}$ Engineering College, Jazan University, Jazan 45142, Saudi Arabia
}

Correspondence should be addressed to Lei Gao; taiyang360@gmail.com

Received 16 November 2014; Revised 15 December 2014; Accepted 16 January 2015

Academic Editor: Fei Dai

Copyright (C) 2015 Lei Gao et al. This is an open access article distributed under the Creative Commons Attribution License, which permits unrestricted use, distribution, and reproduction in any medium, provided the original work is properly cited.

PCC energy pile is a new technology for sustainable development of urban areas. Learning and understanding the temperature variation of PCC energy pile are very important to its development and application. In this study, the Brillouin optical frequency domain analysis (BOFDA) technology is firstly used to measure the temperature variation of PCC energy pile from a model test. The aim is to provide an optical fiber sensing method for monitoring the temperature distribution of PCC energy pile. When the temperatures of circulating water are $70^{\circ} \mathrm{C}, 60^{\circ} \mathrm{C}, 50^{\circ} \mathrm{C}$, and $40^{\circ} \mathrm{C}$, the result shows that the temperatures of PCC energy pile under different conditions are measured well by the optical fiber sensor. It will help to master the temperature distribution and thermomechanical characteristic of PCC energy pile. It can also provide the important scientific and theoretical basis for the design and application of PCC energy pile.

\section{Introduction}

The solar energy and geothermal energy on the surface of earth which are restored in the shallow soil can be absorbed by ground source heat pump which will provide the energy to the building. It is a double system of heating and cooling in the energy-efficient way, less pollution, and environment protection based on the principle of heat pump. For the reasons above, ground source heat pump is widely used in engineering application. In recent years, the underground space is on the fast exploitation. The energy pile technology combined with the ground source heat pump and pile foundation is proposed by the civil engineers. Various shapes of heat exchanger units are laid in the pile foundation. The low temperature geothermal energy can be transferred in the shallow layer soil. It will meet the conventional mechanical function and realize the heat exchange of the shallow geothermal energy. It plays a dual role of pile foundation and ground source heat pump. The cast-in-place concrete large-diameter pipe pile (PCC pile) is a new type of foundation reinforcement technology developed by Hohai University with independent intellectual property rights, which has strong applicability in construction. It is very convenient to use in the soft soil reinforcement and control the quality of construction. Besides, the PCC pile has a very high capacity with low cost and the depth of reinforcement can be up to $25 \mathrm{~m}$ or more. According to the characteristics of PCC pile, a novel energy pile technology called PCC energy pile is developed (see Figure 1), which has the features of efficient circulation of energy, less amount of concrete, high capacity of pile, space saving, and so on. The temperature variation of PCC energy pile is very important to optimize the design and construction of PCC energy pile. However, the little research has been done to the variation of temperature for this new kind of PCC energy pile. Now, the resistive sensor is commonly used to monitor the temperature of pile, but it is very easy to damp and is vulnerable to electromagnetic; it also needs to connect excessive data lines. The data obtained by the resistive sensor is often lost in the project. So the new temperature measurement technology and method should be used to monitor the temperature of PCC energy pile; it will show great significance for the development of PCC energy pile. 
The Brillouin frequency domain analysis (BOFDA) is a new technology for temperature and strain measurement. Using this type of distributed sensing technology, the parameters can be measured as a function of the length optical fiber sensor [1-8]. The strain and temperature distribution information of the entire optical fiber can be continuously measured and real-time display by BOFDA technology. Meanwhile, the optical fiber sensor is a medium and a transmission channel with the characteristics of small size, light weight, geometric shape, and strong adaptability, antielectromagnetic interference, electrical insulation, high sensitivity, and so on. The distributed optical fiber sensor is easy to implement and perform remote monitoring; it can be widely used to monitor the deformation and temperature precisely in geotechnical engineering project [9-13]. In this paper, the new optical fiber sensing technology based on BOFDA is firstly used to monitor the real-time temperature variation of PCC energy pile. The layout of the optical fiber sensor and the PCC energy pile model test are proposed; the temperature data of PCC energy pile are obtained and analyzed.

\section{Principle of BOFDA}

The new distributed optical fiber sensing technology based on BOFDA was proposed in 1996 by Garus and the related experiments were carried out. The accuracy of spatial resolution of $3 \mathrm{~m}$, temperature resolution of $5^{\circ} \mathrm{C}$, and strain resolution of $0.01 \%$ was obtained [2]. The transfer function based on the location detection of the measuring point is achieved; its complex amplitude of the probe and pump light transmission are in full consideration. The temperature and strain distribution of optical fiber sensor can be obtained by calculating the impulse response function.

A fully distributed system can be realized by using the phenomenon called stimulated Brillouin scattering (SBS) in silica optical fiber. The SBS can be seen as the interaction of two counter propagating light waves with an acoustic wave within the optical fiber. There will be a frequency shift of the backscattered light and it depends on the strain and temperature distribution along the optical fiber sensor. The BOFDA analyzer calculates the complex transfer function by comparing with the optical signals in amplitude and phase. This function is converted by the inverse fast Fourier transform. In Figure 2, a bunch of narrow-line width continuous pump light is injected into a single-mode optical fiber sensor from one side and a bunch of narrow-line width continuous probe light is injected into the optical fiber from the other side. The frequency of the probe light is adjusted to a value that is lower than that of the pump light. The frequency difference between them is approximately equal to the Brillouin frequency shift. A variable frequency $f_{m}$ with a sinusoidal signal is to detect the amplitude of modulated light. The detector is used to determine the intensity value of the signal at each frequency of probe light and pump light. The output signal of detector is input to the network analyzer instrument that is based on the light baseband transmission function. The output signal $h(t)$ of network analyzer converted by the inverse fast Fourier transform

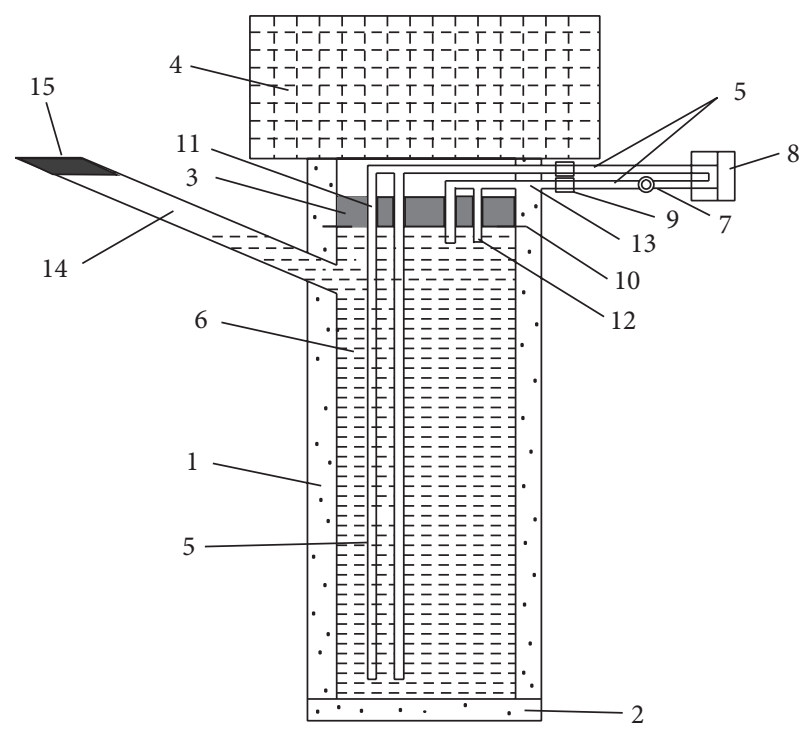
(1) PCC pile
(9) Removable contact
(2) Base plate
(10) Dowel
(3) Cover plate
(11) Inlet hole
(4) Superstructure
(12) Out hole
(5) Heat pipe
(13) Preformed hole
(6) Thermal fluid
(7) Geothermal heat pump
(14) Check passage
(15) Seal plate

(8) Collectors

Figure 1: Design of PCC energy pile [14].

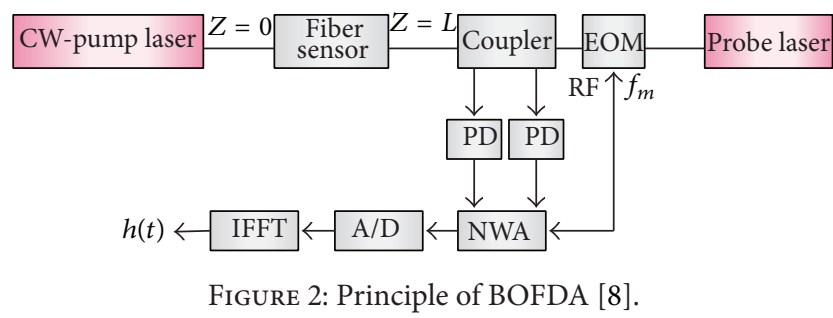

contains the strain or temperature distribution information along the optical fiber sensor [6-8]. Figure 2 shows the principle of BOFDA system.

\section{Model Test of PCC Energy Pile}

3.1. Instrument. The BOFDA instrument used in this study is fTB2505 produced by a German company called fibrisTerre $\mathrm{GmbH}$. The instrument can be used for the continuous, long-distance strain and temperature monitoring by the optical fiber. It is also used to monitor the early damage of geotechnical structures, fatigue strength of bridges, tunnels, dams, high-precision strain of oil pipeline, and so on. The fTB2505 has the measure accuracy of less than $2 \mu \varepsilon$ and temperature measurement accuracy of less than $0.1^{\circ} \mathrm{C}$. It can achieve the spatial resolution of $0.5 \mathrm{~m}$ within the range of 


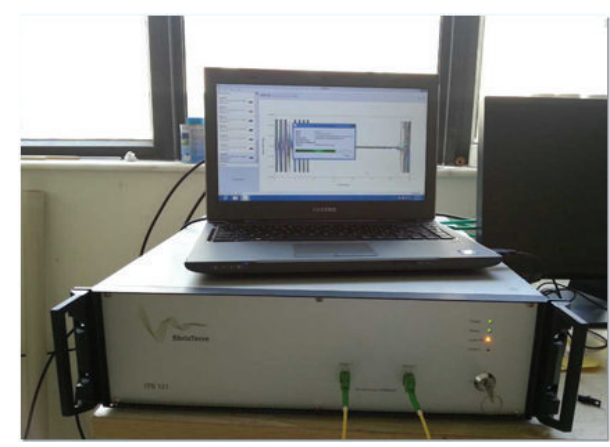

(a) Instrument

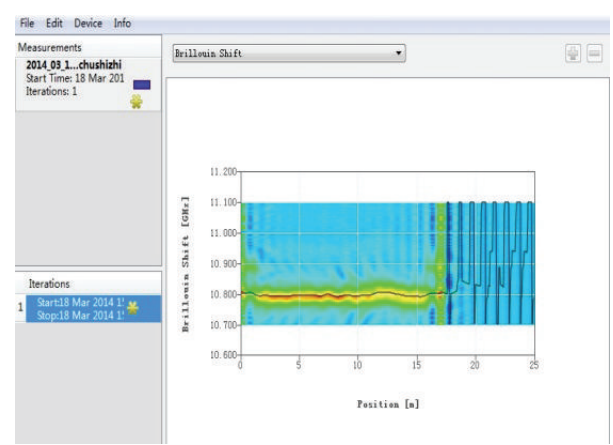

(b) Software

Figure 3: Test instrument and software.

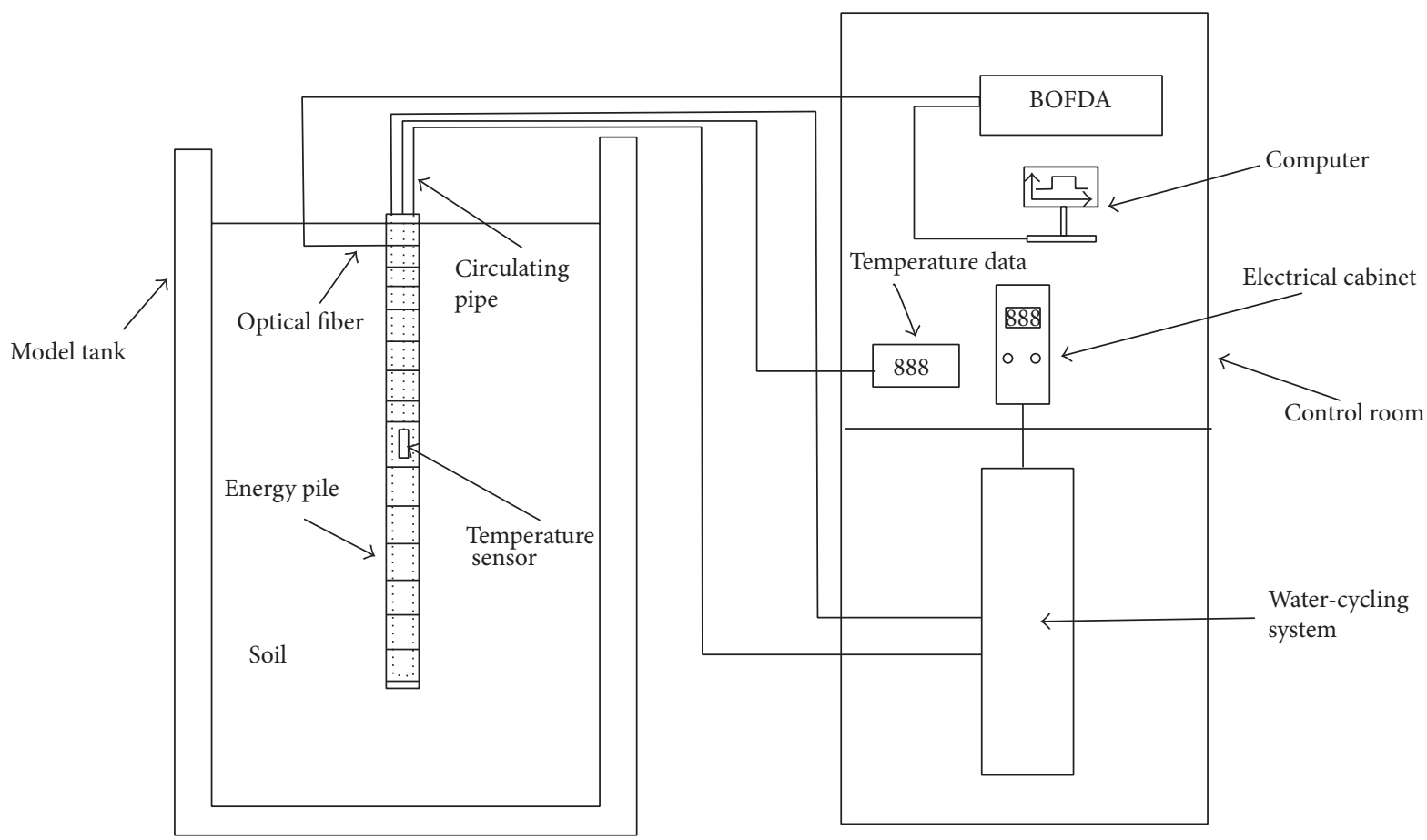

Figure 4: Diagram of model test for PCC energy pile.

$25 \mathrm{~km}$. The parameters of BOFDA with the type of fTB2505 are shown in Tables 1 and 2.

The fTB2505 is a readout unit for the distributed temperature and strain measurement using the optical fiber sensor. It can realize long-range, continuous sensing and structural health monitoring, which is very useful for the civil and geotechnical engineering project. The measurement system includes the monitoring software FTView, FTB readout unit, and a computer. The instrument and software can be seen in Figure 3.

3.2. Test Equipment. In order to simulate the actual work state of PCC energy pile, a PCC energy pile model test based on BOFDA is proposed. The test equipment mainly includes a PCC energy pile, soil, a heating device, and an optical sensing fiber sensor. Figure 4 is the diagram of model test for PCC
TABLE 1: Parameters of fTB2505.

\begin{tabular}{lccc}
\hline Label & Parameters & Label & Parameters \\
\hline $\begin{array}{l}\text { Fiber } \\
\text { model }\end{array}$ & $\begin{array}{c}\text { Standard } \\
\text { single-mode } \\
\text { optical fiber }\end{array}$ & Distance & $25 \mathrm{~km}$ \\
\hline $\begin{array}{l}\text { Connector } \\
\text { type }\end{array}$ & E-2000/APC & $\begin{array}{c}\text { Spatial } \\
\text { resolution }\end{array}$ & $0.5 \mathrm{~m}$ \\
\hline $\begin{array}{l}\text { Detection } \\
\text { form }\end{array}$ & $\begin{array}{c}\text { Double-end } \\
\text { detection }\end{array}$ & $\begin{array}{c}\text { Monitoring } \\
\text { mode }\end{array}$ & $\begin{array}{c}\text { Single, } \\
\text { continuous, } \\
\text { custom }\end{array}$ \\
\hline $\begin{array}{l}\text { Spatial } \\
\text { accuracy }\end{array}$ & $0.05 \mathrm{~m}$ & Size & $L \times W \times H \begin{array}{r}495 \times 482 \\
\times 145\end{array}$ \\
\hline
\end{tabular}

energy pile based on BOFDA. The model can also be used to study the temperature variation of different type of energy pile. The size of model tank used in the test is $2 \mathrm{~m} \times 1 \mathrm{~m} \times 1.5 \mathrm{~m}$. 
TABLE 2: Indices of fTB2505.

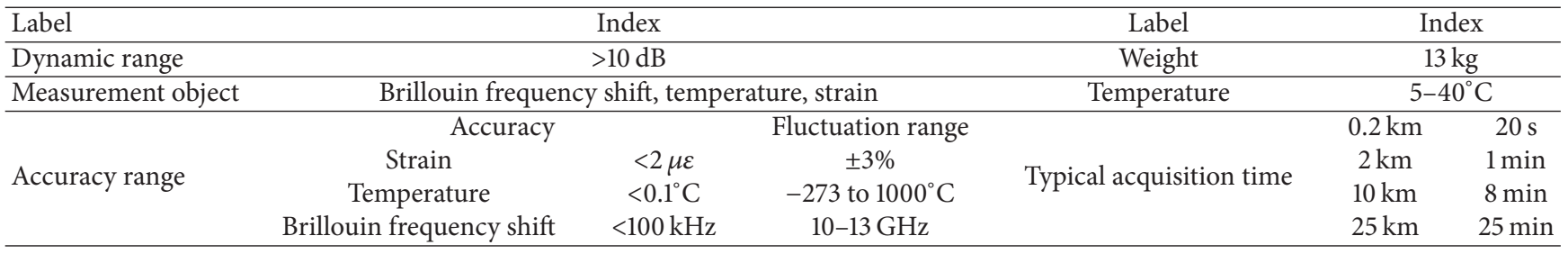

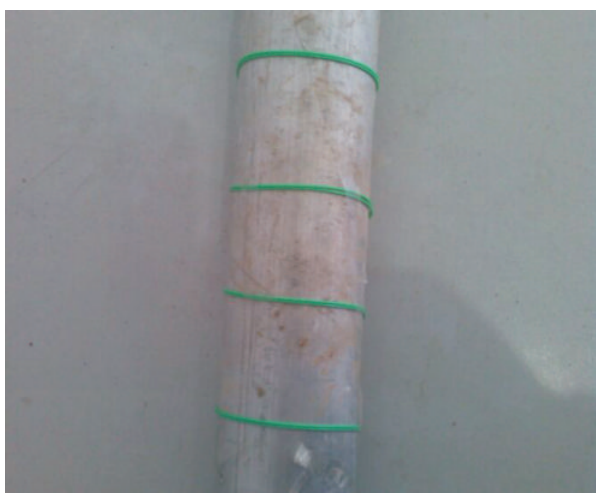

(a) Installation of optical fiber sensor

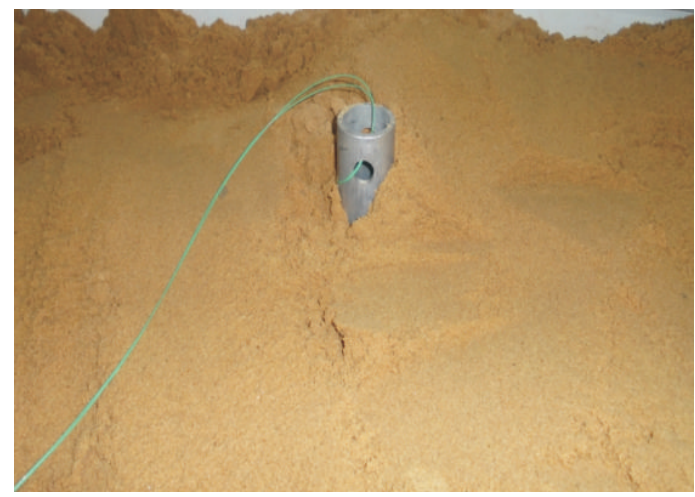

(b) Pile and soil

Figure 5: Photos of model test for PCC energy pile.

The heater provides the heat to the model of PCC energy pile. The heater consists of four parts: thermostat control panel, heating pipe, pump, and circulating water. The heater uses circulating hot water as heat transfer medium; the water is heated by using electricity. It has a touch control electric cabinet which is easy to operate. Meanwhile, it also has the characteristics of fast heating and cooling, high accuracy, stability, and unique heat design with power. The single-mode optical fiber sensor is used as the temperature sensor in this test shown in Figure 5(a). The optical fiber sensor responds quickly and can be monitored remotely to the long distance which can ensure the safety of person and equipment in the project. The optical sensing fiber sensor is laid helically as shown in Figure 5(b); it should be examined in case there is some damage produced during the installation process.

3.3. Result and Discussion. The model of PCC energy pile has the diameter of $5 \mathrm{~cm}$ and the length of $1.0 \mathrm{~m}$. An optical fiber sensor is fixed along the pile. The temperatures of circulating water are $70^{\circ} \mathrm{C}, 60^{\circ} \mathrm{C}, 50^{\circ} \mathrm{C}$, and $40^{\circ} \mathrm{C}$; the temperatures of PCC energy pile under different conditions are measured. It can be seen in Figure 6 that the temperature of the optical fiber sensor along with PCC energy pile can be obtained during the measurement time that lasts for 180 seconds, and the temperature of the optical fiber sensor shows a decreasing trend with time. The temperature distribution of PCC energy pile with $70^{\circ} \mathrm{C}, 60^{\circ} \mathrm{C}, 50^{\circ} \mathrm{C}$, and $40^{\circ} \mathrm{C}$ is shown in Figures 6(a), 6(b), 6(c), and 6(d), respectively. It indicates that the temperature of PCC energy pile decreases when the water temperature decreases. When the water temperature decreases to $40^{\circ} \mathrm{C}$ which is equal to the temperature of PCC energy pile, there will be very small variation of temperature for the pile. The results indicate that the model test of PCC energy pile based on BOFDA is successfully developed to simulate the working state and monitor the temperature variation of PCC energy pile. The optical sensing fiber sensor is used to obtain a lot of useful data and good results for the pile.

PCC energy pile is a new pile; the reinforcement cost of PCC energy pile is low and very close to a flexible pile, while it has an effect of rigid reinforcement. The PCC energy pile can be used in the clay, sand, and silt soil area. In order to master the temperature distribution and thermomechanical characteristics of PCC energy pile, a PCC energy pile model test based on BOFDA is developed; the model test system is very good for the testing. The temperature of PCC energy pile is often measured by the resistive sensor. But the resistive sensor is easily destroyed; it also needs to connect excessive data lines and have only one piece of data for each monitoring point; the data is often lost in the project; it cannot obtain all the temperature information of PCC energy pile. So the new BOFDA technology is used to monitor the temperature of PCC energy pile in this test system; the result shows that the BOFDA technology can measure the temperature of PCC energy pile well and the optical fiber sensor is used to measure the temperature of every point for the pile. In the future, the strain of PCC energy pile should also be measured by the optical fiber sensor; this will show great significance for the development of PCC energy pile.

\section{Conclusions}

(1) PCC energy pile is a new type of pile, which has the advantages of energy saving and environmental 


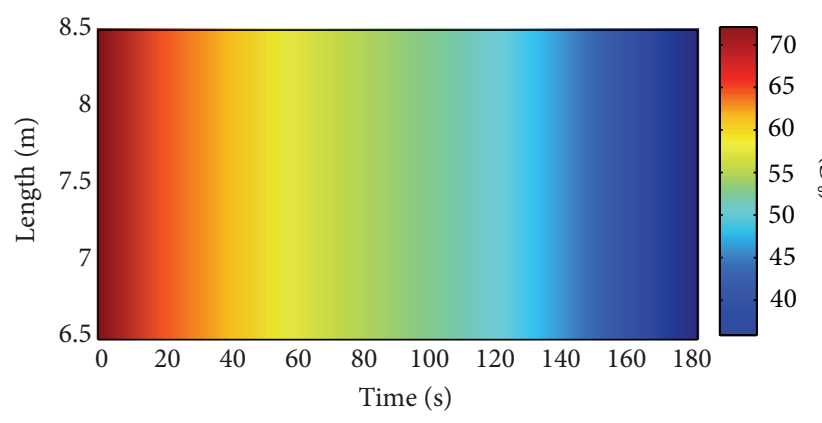

(a)

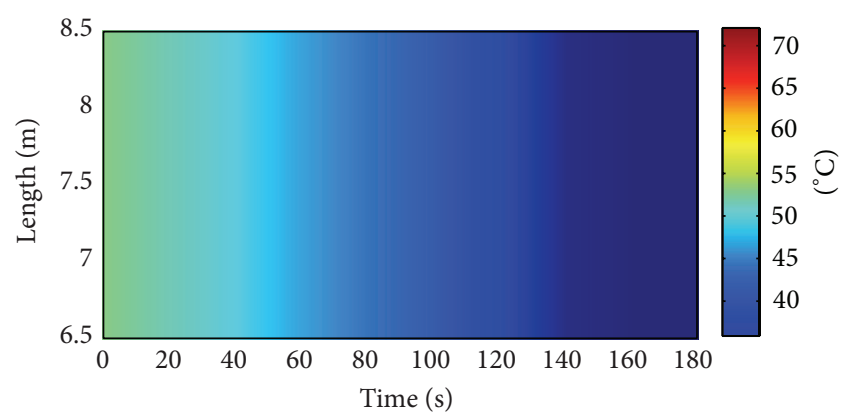

(c)

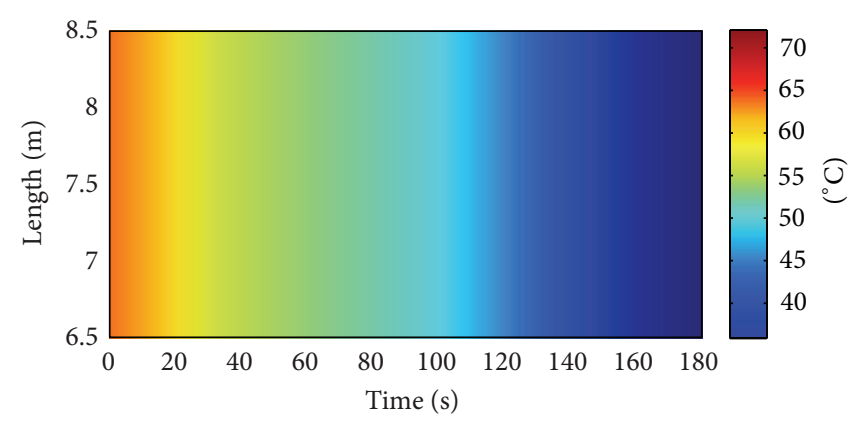

(b)

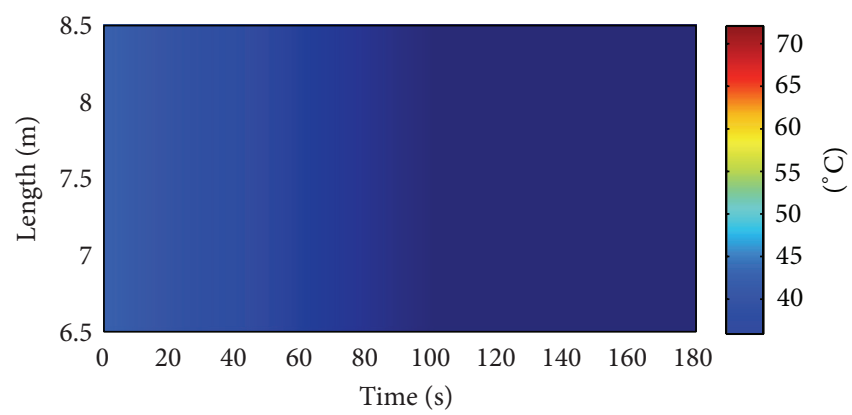

(d)

FIGURE 6: Variation of temperature for PCC energy pile.

protection. The cost of PCC energy pile is low; it can be used in the clay, sand, and silt soil area and will have a very broad prospect.

(2) As a new technology, the BOFDA monitoring technology is firstly used to measure the temperature of PCC energy pile; it shows that the optical fiber has the advantages of simple layout, high survival rate, and high precision.

(3) A PCC energy pile model test based on BOFDA is developed. The temperature variation of PCC energy pile can be effectively monitored by using BOFDA technology; the temperature information of PCC energy pile is obtained and the model test achieves good results.

\section{Conflict of Interests}

The authors declare that there is no conflict of interests regarding the publication of this paper.

\section{Acknowledgments}

Financial supports from the Fundamental Research Funds for the Central Universities of Hohai University (no. 2014B04914), China Postdoctoral Science Foundation (nos. 2012M511193 and 2014T70468), Ph.D. Programs Foundation of Ministry of Education of China (no. 20120094120015), the Natural Science Foundation of Jiangsu Province (no. BK20130832), and the National Natural Science Foundation of China (no. 51378178) are gratefully appreciated.

\section{References}

[1] D. Garus, T. Gogolla, K. Krebber, and F. Schliep, "Brillouin optical-fiber frequency-domain analysis for distributed temperature and strain measurements," Journal of Lightwave Technology, vol. 15, no. 4, pp. 654-662, 1997.

[2] R. Bernini, A. Minardo, and L. Zeni, "An accurate highresolution technique for distributed sensing based on frequency-domain brillouin scattering," IEEE Photonics Technology Letters, vol. 18, no. 1, pp. 280-285, 2006.

[3] X. Bao and L. Chen, "Recent progress in distributed fiber optic sensors," Sensors, vol. 12, no. 7, pp. 8601-8639, 2012.

[4] F. Wang, C. Li, X. Zhao, and X. Zhang, "Using a Mach-Zehnderinterference-based passive configuration to eliminate the polarization noise in Brillouin optical time domain reflectometry," Applied Optics, vol. 51, no. 2, pp. 176-180, 2012.

[5] X. Zhang, Y. Lu, F. Wang, H. Liang, and Y. Zhang, "Development of fully-distributed fiber sensors based on brillouin scattering," Photonic Sensors, vol. 1, no. 1, pp. 54-61, 2011.

[6] Y. Yao, Y. Lu, X. Zhang, F. Wang, and R. Wang, "Reducing trade-off between spatial resolution and frequency accuracy in BOTDR using cohen's class signal processing method," IEEE Photonics Technology Letters, vol. 24, no. 15, pp. 1337-1339, 2012.

[7] R. Bernini, A. Minardo, and L. Zeni, "Distributed sensing at centimeter-scale spatial resolution by BOFDA: measurements and signal processing," IEEE Photonics Journal, vol. 4, no. 1, pp. 48-56, 2012.

[8] C. A. Galindez-Jamioy and J. M. López-Higuera, "Brillouin distributed fiber sensors: an overview and applications," Journal of Sensors, vol. 2012, Article ID 204121, 17 pages, 2012.

[9] H.-H. Zhu, A. N. L. Ho, J.-H. Yin, H. W. Sun, H.-F. Pei, and C.-Y. Hong, "An optical fibre monitoring system for evaluating 
the performance of a soil nailed slope," Smart Structures and Systems, vol. 9, no. 5, pp. 393-410, 2012.

[10] Y. Lu, B. Shi, G. Q. Wei, S. E. Chen, and D. Zhang, "Application of a distributed optical fiber sensing technique in monitoring the stress of precast piles," Smart Materials and Structures, vol. 21, no. 11, Article ID 115011, 2012.

[11] X. Weng, H.-H. Zhu, J. Chen, D. Liang, B. Shi, and C.-C. Zhang, "Experimental investigation of pavement behavior after embankment widening using a fiber optic sensor network," Structural Health Monitoring, vol. 14, no. 1, pp. 46-56, 2015.

[12] H.-H. Zhu, B. Shi, J.-F. Yan, J. Zhang, C.-C. Zhang, and B.J. Wang, "Fiber Bragg grating-based performance monitoring of a slope model subjected to seepage," Smart Materials and Structures, vol. 23, no. 9, Article ID 095027, 2014.

[13] H.-H. Zhu, B. Shi, J. Zhang, J.-F. Yan, and C.-C. Zhang, "Distributed fiber optic monitoring and stability analysis of a model slope under surcharge loading," Journal of Mountain Science, vol. 11, no. 4, pp. 979-989, 2014.

[14] H. L. Liu, G. Q. Kong, and W. W. N. Charles, "Applications of energy piles and technical development of PCC energy piles," Chinese Journal of Geotechnical Engineering, vol. 36, no. 1, pp. 176-181, 2014. 

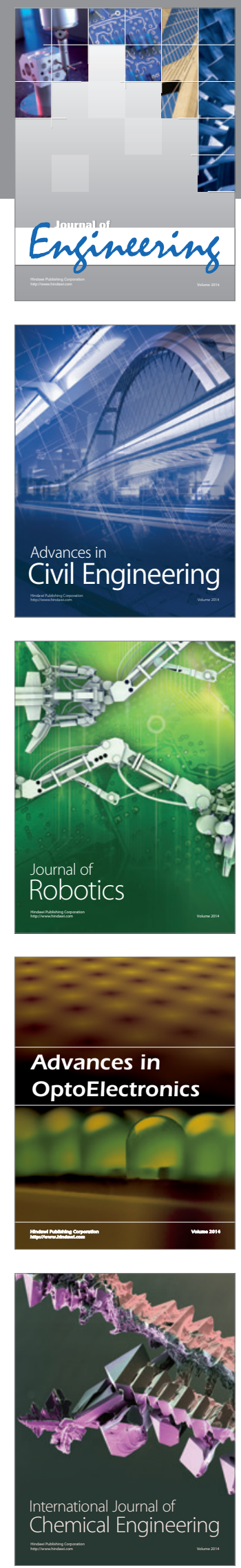

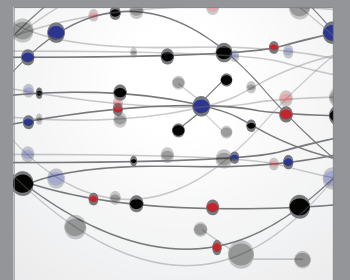

The Scientific World Journal
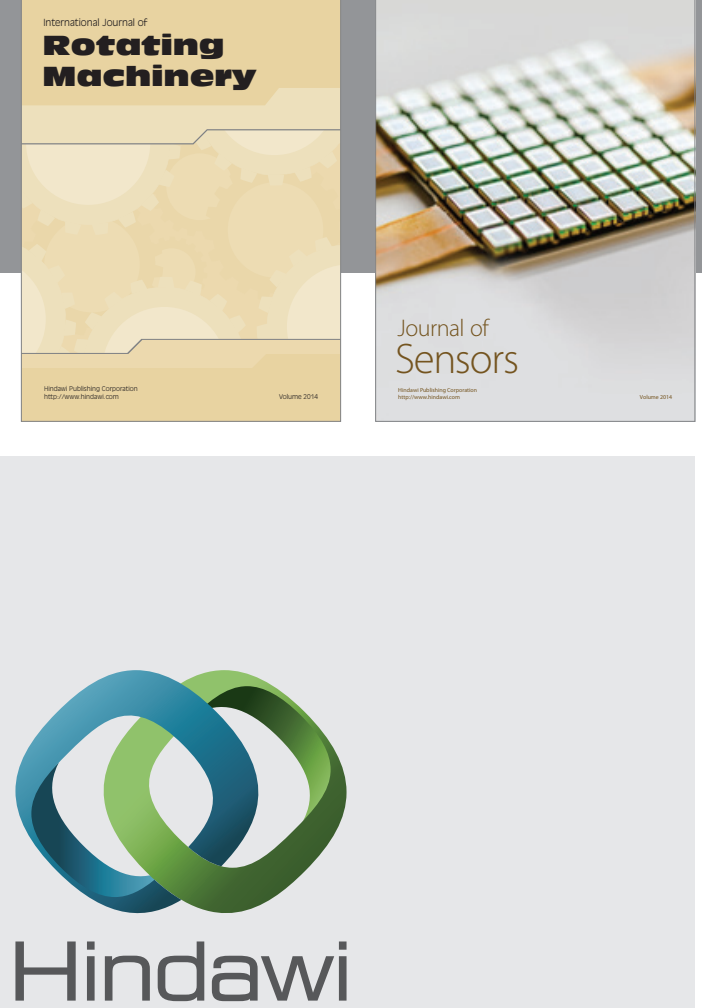

Submit your manuscripts at http://www.hindawi.com
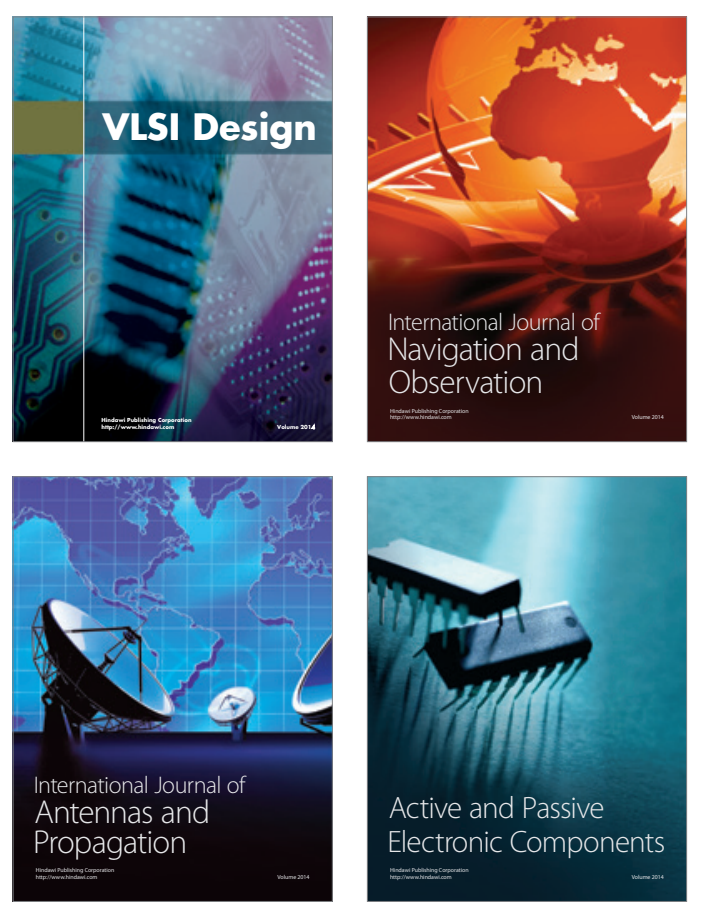
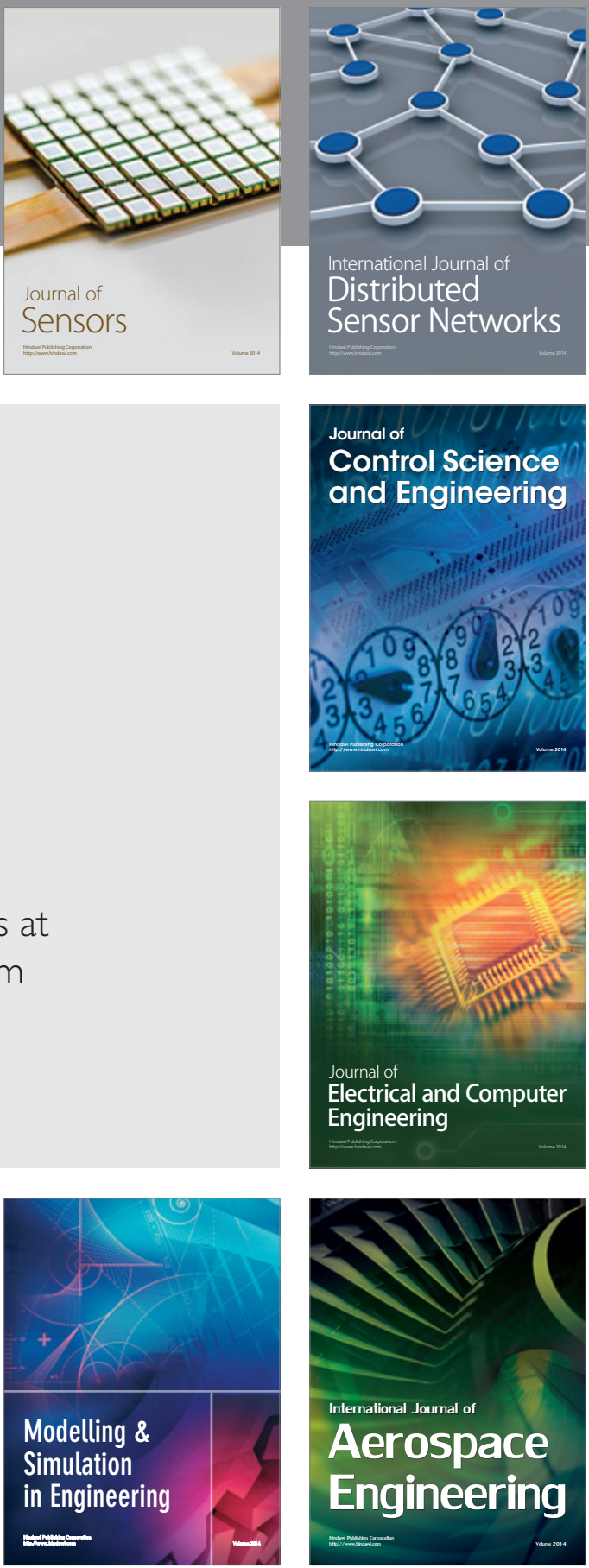

Journal of

Control Science

and Engineering
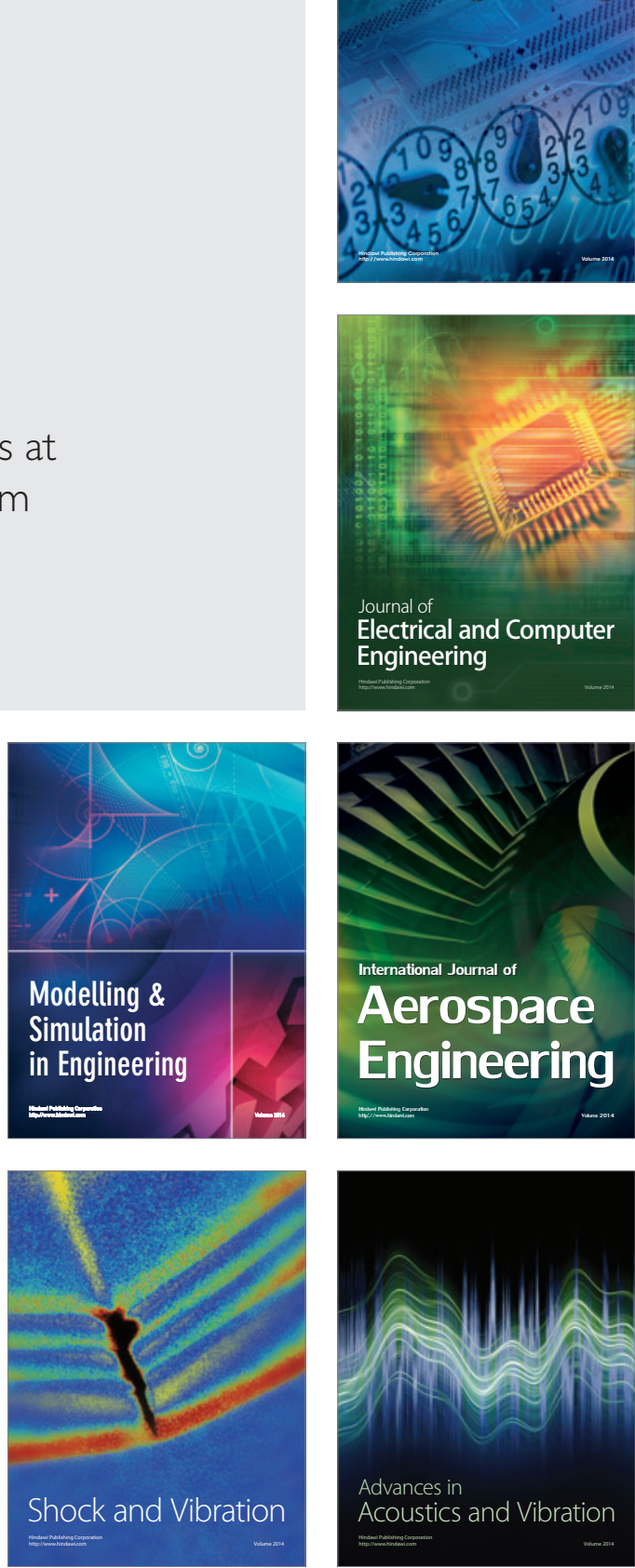